educational improvements: recognition of incidence of TD associated with different antipsychotic therapies, differentiation of TD from parkinsonism, and the personalized selection of therapies for the management of TD. $37 \%$ of psychiatrists had a measurable increase in confidence in understanding the role of the interprofessional team in recognizing TD after activity participation.

Conclusions. The results indicated that a CME-certified 30-minute video activity was effective at improving knowledge among psychiatrists for the recognition and management of TD. Future education should continue to address best practices in the care of patients with TD.

Funding. Neurocrine Bioscience, Inc

\section{Characterization and Treatment Goals of Patients on Long-Acting Injectable vs Oral Antipsychotics: Results from a Patient/Caregiver/ Psychiatrist Survey}

Heather M. Fitzgerald, PharmD ${ }^{1}$, Jason Shepherd, BA $^{2}$, Hollie Bailey, $\mathrm{BSc}^{2}$, Mia Berry, $\mathrm{BSc}^{2}$, Jack Wright, $\mathrm{MSc}^{2}$ and Maxine Chen, $\mathrm{PhD}^{3}$

${ }^{1}$ Lundbeck LLC, Deerfield, IL, USA, ${ }^{2}$ Adelphi Real World, Bollington, Cheshire, United Kingdom, and ${ }^{3}$ Otsuka Pharmaceutical Development \& Commercialization, Inc., Princeton, NJ, USA

Presenting Author: Heather M. Fitzgerald

\section{Abstract}

Background. Patient preferences in schizophrenia (SCZ), including identification of key goals and outcomes for treatment and relative importance of certain treatment goals to patients, have been assessed by several studies. However, there continues to be a lack of sufficient evidence on US patient attitudes and perceptions towards treatment goals and pharmacotherapy options in SCZ, especially taking into context long-acting injectable antipsychotics (LAIs) in this disease area. This lack of evidence is further pronounced in caregivers of individuals with SCZ. The objective of this analysis was to characterize patients with SCZ on LAIs vs patients on oral antipsychotics (OAPs) and evaluate the treatment goals of patients in each group.

Methods. This was a real-world, cross-sectional survey of US psychiatrists, patients $=18$ years old with a diagnosis of SCZ, and caregivers. Data was collected using the Disease Specific Programme (DSP) methodology, which has been previously published. Psychiatrists $(n=120)$ completed detailed record forms for next 8 consecutive outpatients and 2 inpatients matching inclusion criteria, including non-interventional clinical and subjective assessments. The same patients and their caregivers, if present, were invited by their psychiatrist to voluntarily complete a separate survey.

Results. Of 1135 patients on treatment where the physician provided survey data; 251 were on an LAI, and 884 were on an OAP. Mean (SD) time to SCZ diagnosis for those on an LAI was 10.3 (12.0) years vs 7.8 (10.5) years for those on OAPs. More patients in the LAI vs OAP group were being treated as an inpatient $(27.1 \%$ vs $15.7 \%$, respectively; $\mathrm{p}<0.0001$ ). Patients on an LAI reported being on their current medication regimen for less time (mean 1.7 years) vs those on OAPs (mean 2.5 years) ( $\mathrm{p}=0.0093)$. More patients on LAIs were unemployed due to disability vs those on OAPs (56.1\% vs $39.5 \%$, respectively), and less patients on LAIs were able to work part-time or full-time ( $21.1 \%$ or $4.1 \%)$ vs those on OAPs $(23.2 \%$ or $11.4 \%)$. More patients on an LAI had a caregiver vs those on OAPs ( $37.3 \%$ vs $26.1 \%$, respectively; $\mathrm{p}=0.0011)$. Regarding the most important treatment goals reported by patients, both groups reported similar preferences for decrease in disease symptoms (62\% on LAI vs $65 \%$ on OAPs) and thinking more clearly (53\% on LAI vs $46 \%$ on OAPs); however, a numerically higher proportion of LAI patients reported that the current medication helped decrease hospitalizations due to relapse vs those on OAPs ( $38 \%$ vs $32 \%$, respectively).

Discussion. Given the characteristics of patients participating in this real-world survey, those on LAIs exhibited qualities which indicate a higher severity of illness vs those on OAPs. Results suggest that treatment with LAIs is still mainly being provided to patients later in the disease course and/or who have adherence problems, despite a growing body of evidence of utility in younger patients earlier in the course of illness.

Funding. Otsuka Pharmaceutical Development \& Commercialization, Inc. and Lundbeck LLC

\section{A Novel Real-Time PCR Assay for Detection of HLA-A*31:01 in Individuals Being Considered for Carbamazepine Therapy}

David S. Krause, MD, Kathleen Davis, BS, Daniel Dowd, PharmD and David J. Robbins, PhD

Genomind, Inc., King of Prussia, PA, USA

Presenting Author: David S. Krause

\begin{abstract}
Background. Carbamazepine, an anticonvulsant also used as a mood stabilizer and for trigeminal neuralgia, is associated with serious, sometimes fatal cutaneous adverse drug reactions, including Stevens Johnson Syndrome and toxic epidermal necrolysis1. Current literature demonstrates a genetic predisposition linked to specific class I and II human leukocyte antigen (HLA) types in various ethnic populations 2 . HLA-A ${ }^{\star} 31: 01$ is one such HLA type, and is routinely identified by the tag SNP rs1061235. However, rs1061235 has poor specificity for HLA*31:01 due to interference of HLA-A ${ }^{\star} 33$ types3. We investigated the false positive rate in our population and developed a novel real-time PCR assay that distinguishes HLA-A ${ }^{\star 31: 01}$ from other HLA-A types including HLA-A ${ }^{\star} 33$.

Methods. 120 unique samples were tested in triplicate during the validation of this assay and were sent to a reference lab for HLA next generation sequencing (NGS) typing, including 89 in-house samples and 31 Coriell samples with documented HLA typing results. The results from our real-time PCR assay were compared
\end{abstract}


to the HLA typing results. HLA typing results were also compared to the tag SNP rs1061235 results to calculate the false positive rate. Results. There was $100 \%$ concordance between our real-time PCR results and expected results based on HLA typing. 89 sample results for tag SNP rs1061235 were compared to HLA typing results. 75/89 samples had a rs 1061235 variant, but $31 / 75(41 \%)$ samples did not have the HLA-A*31:01 type, thus defining the false positive rate of the tag SNP for our population. We theorized there would be a small subset of rare HLA-A types that would interfere with the assay and we tested the three types available to us. We confirmed that 3 of the HLA types (HLA-A*31:04, 31:12, and 31:16) result falsely positive due to sequence homology with $31: 01$. There is no known literature indicating whether these rare HLA-A*31 subtypes are associated with cutaneous adverse reactions. These 3 HLA types and the other suspected interfering HLA types have limited frequency data sets and are expected to occur rarely in our patient population; we expect these HLA types make up less than $0.003 \%$ of the our population. Our assay specificity for the validation is $>99 \%$. Conclusions. Our custom real-time PCR assay for detection of HLA-A ${ }^{\star} 31: 01$ is significantly more specific than the commonly used tag SNP rs1061235. Clinicians considering carbamazepine therapy for their patients will have a better understanding of cutaneous adverse reaction risk and can make improved personalized treatment decisions. This quick, cost effective assay allows more patients in need of carbamazepine treatment to benefit from its use. Funding. Genomind, Inc.

\section{Can Low Dose Sertraline Cause Serotonin Syndrome in Pediatric Patients? 2 Case Reports}

Sultana Jahan, MD

University of Missouri, Department of Psychiatry, Columbia, MO, USA

Presenting Author: Sultana Jahan

\begin{abstract}
Background. Serotonin syndrome is a potentially life-threatening condition associated with increased serotonergic activity in the central nervous system. Serotonin syndrome is underreported complication of pharmacotherapy. The Hunter Criteria for serotonin syndrome (SS) are fulfilled if the patient has taken a serotonergic agent and has one of the following symptoms: 1) spontaneous clonus, 2) inducible clonus and agitation or diaphoresis, 3) ocular clonus and agitation or diaphoresis, 4) tremor and hyperreflexia, 5) hypertonia, or 6) temperature above $38 \mathrm{C}$ and ocular clonus or inducible clonus.

Method. Patient A was a 16-year-old Caucasian male with history of major depressive disorder, social anxiety and OCD who presented to the emergency room with multiple complaints: twitching of bilateral cheeks, intermittent tremor of his hands and feet, mental fogginess/confusion, stuttering when attempting to speak, agitation, profuse sweating and headache. 3 weeks prior, his sertraline dose was increased from $25 \mathrm{mg}$ daily to $50 \mathrm{mg}$ daily. His physical exam was remarkable for elevated blood pressure and
\end{abstract}

heart rate as well as hyperreflexia noted on patellar reflex testing. No significant abnormalities were noted on routine labs. He was told his symptoms were likely due to medication side effects. The patient was discharged with instructions to decrease his sertraline dose from $50 \mathrm{mg}$ to $25 \mathrm{mg}$ daily and follow up with his outpatient psychiatrist. 2 days later the patient was seen at the outpatient child psychiatry clinic and he was advised to taper off sertraline completely by taking $12.5 \mathrm{mg}$ daily for 3 days before cessation. After stopping the medication, the patient's symptoms resolved. Patient B was a 16-year-old female with generalized anxiety disorder and major depressive disorder who presented to the general pediatric clinic with progressively worsening hand tremors and body shaking since her Zoloft dose was increased from $25 \mathrm{mg}$ to $50 \mathrm{mg}$ daily. She also felt it was more difficult to hold objects. At the physical exam she had an elevated heart rate to 93 and elevated blood pressure to $182 / 75$. Her deep tendon reflexes were $4+$ bilaterally. Upon consultation with child psychiatry, the patient was recommended to taper off sertraline. After the discontinuation of sertraline, her symptoms resolved.

Result. These 2 patients developed mild to moderate symptoms of serotonin syndrome with low doses of sertraline. Symptoms resolved after the discontinuation of the SSRI.

Discussion. In the pediatric patient population, serotonin syndrome can develop even with lower doses of an SSRI. To avoid a missed diagnosis, clinicians should familiarize themselves with the Hunter Criteria for serotonin syndrome. It is also vital to educate parents and caregivers about the toxicities of SSRIs, including serotonin syndrome, so they may monitor treatment and take appropriate action if needed.

\section{Phase 3 Safety and Tolerability Results of the Combination Olanzapine and Samidorphan in Patients with Schizophrenia: The 1 Year ENLIGHTEN-2-Extension}

\author{
Rene Kahn, $\mathrm{MD}, \mathrm{PhD}^{1}$, Bernard Silverman, $\mathrm{MD}^{2}$, \\ Lauren DiPetrillo, $\mathrm{PhD}^{2}$, Christine Graham, $\mathrm{PhD}^{2}$, \\ Ying Jiang, $\mathrm{PhD}^{2}$, Jiani Yin, $\mathrm{PhD}^{2}$, Adam Simmons, $\mathrm{MPH}^{2}$, \\ Vasudev Bhupathi, $\mathrm{MS}^{2}$, Bei $\mathrm{Yu}, \mathrm{MD}, \mathrm{PhD}^{2}$, \\ Craig Hopkinson, $M^{2}$ and Davidd McDonnell, $M^{3}$
}

${ }^{1}$ Icahn School of Medicine at Mount Sinai, New York, NY, USA, ${ }^{2}$ Alkermes, Inc., Waltham, MA, USA, and ${ }^{3}$ Alkermes Pharma Ireland Limited, Dublin, Ireland

\section{Presenting Author: Adam Simmons}

\section{Abstract}

Objective. Combination olanzapine and samidorphan (OLZ/SAM) is in development for treatment of schizophrenia and bipolar I disorder and is intended to provide the antipsychotic efficacy of olanzapine while mitigating olanzapine-associated weight gain. This 52-week open-label extension study (NCT02873208; ENLIGHTEN2-EXT) in schizophrenia assessed the safety and tolerability of OLZ/SAM. Methods: Patients completing the 24-week, randomized, 